DE

M E D I C I N A

T R O P I C A L

$\mathrm{DE}$

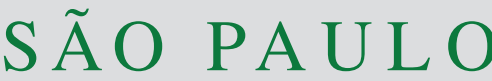

JOURNAL OF THE SÃO PAULO INSTITUTE OF TROPICAL MEDICINE

(1)Corporación para Investigaciones Biológicas (CIB), Medical and Experimental Mycology Group, Medellín, Colombia

(2)Centers for Disease Control and Prevention, Mycotic Diseases Branch, Atlanta, United States

(3) Universidad de Antioquia, Escuela de Microbiología, Grupo de Investigación en Microbiología Básica y Aplicada, Medellín, Colombia

(4)Universidad del Rosario, Escuela de Medicina y Ciencias de la Salud, Bogotá, Colombia

Correspondence to: Beatriz L. Gómez Corporación para Investigaciones Biológicas, Grupo de investigación en Micología Médica y Experimental, Carrera 72A \# 78B-141, Medellín, Colombia Tel: +5746051808 , Fax: +5746051808

E-mail: beatrizlgomez@ hotmail.com

Received: 5 May 2017

Accepted: 10 August 2017

\section{Evaluation of a Cryptococcal antigen Lateral Flow Assay in serum and cerebrospinal fluid for rapid diagnosis of cryptococcosis in Colombia}

Diego H. Cáceres ${ }^{1}$, Alejandra Zuluaga' ${ }^{1}$, Ángela M. Tabares ${ }^{1}$, Tom Chiller², Ángel González ${ }^{3}$, Beatriz L. Gómez ${ }^{1,4}$

\section{ABSTRACT}

A Lateral Flow Assay to detect cryptococcal antigen ( $\left.\mathrm{CrAg}^{\circledR} \mathrm{LFA}\right)$ in serum and cerebrospinal fluid for the rapid diagnosis of cryptococcosis was evaluated. A retrospective validation was performed. Sensitivity and specificity of the $\mathrm{CrAg}^{\circledR}$ LFA was $100 \%$. High concordance (kappa index=1.0) between Cryptococcal Antigen Latex Agglutination System $\left(\mathrm{CALAS}^{\circledR}\right)$ and $\mathrm{CrAg}^{\circledR}$ LFA was observed. $\mathrm{CrAg}^{\circledR}{ }^{\circledR}$ LFA showed higher analytical sensitivity for detecting low concentrations of cryptococcal antigen.

KEYWORDS: Cryptococcosis. Cryptococcus. Antigen. Diagnosis. Point-of-care. Lateral Flow Assay. Immunochromatographic assay. Latex agglutination system

Cryptococcal meningitis primarily occurs in AIDS patients and especially in those with CD4 cell counts less than 100 cells/ $\mu \mathrm{L}$, and continues causing high mortality and morbidity, especially in resource-limited countries ${ }^{1}$. In Colombia, as in other Latin American countries, access to antiretroviral therapy (ART) may be limited. As a result, cryptococcosis and other opportunistic infections (OIs) are major causes of mortality in people living with HIV and AIDS (PLWHA) ${ }^{1}$. Early and rapid diagnosis of cryptococcosis reduces the risk of mortality and morbidity and the development of severe disease by facilitating prompt and timely treatment ${ }^{2}$. Detection of cryptococcal antigen in serum and cerebrospinal fluid (CSF) has proven to be a highly sensitive (93-100\%) and specific (93-98\%) method for diagnosing cryptococcosis ${ }^{3}$. In this study, we compared two cryptococcal antigen tests, one dipstick sandwich immunochromatographic assay developed by IMMY ${ }^{\circledR}$ (Immuno-Mycologics, Norman, Oklahoma, USA) and a Cryptococcal Antigen Latex Agglutination System $\left(\mathrm{CALAS}^{\circledR}\right)$ developed by Meridian Bioscience (Cincinnati, Ohio, USA).

A retrospective validation study of the $\mathrm{CrAg}^{\circledR}$ LFA test was conducted in the Medical Mycology laboratory of a private research institution, the Corporación para Investigaciones Biológicas (CIB), in Medellín, Colombia. Serum and CSF samples from patients with or without diagnosis of cryptococcosis were randomly selected from a collection of biological samples stored in the CIB's biobank. All samples were coded and analyzed blindly. Diagnosis of cryptococcosis was based on the EORTC/MSG definitions ${ }^{4}$. A case was considered proven if Cryptococcus was isolated from blood, tissue, sterile fluids or respiratory specimens, or if there was a positive antigen test in serum or CSF using CALAS ${ }^{\circledR}{ }^{4}$. Sensitivity and specificity of the $\mathrm{CrAg}^{\circledR}$ LFA were calculated using microbiological criteria for diagnosing cryptococcosis. The capacity to perform the diagnosis of cryptococcosis 
between $\mathrm{CrAg}^{\circledast}$ LFA and $\mathrm{CALAS}^{\oplus}$ was compared, and the ability to quantify Cryptococcus antigens in CSF was also evaluated for each test ${ }^{5}$. Positive antigen samples were diluted initially $1: 4$, followed by a two-fold serial dilution up to $1: 16,184$ (dilutions are shown in Figure 1). $\mathrm{CrAg}^{\circledR}$ LFA and CALAS ${ }^{\circledR}$ were performed in parallel using the same sample's dilutions.A total of $51 \mathrm{CSF}$ and 32 serum samples from 83 patients were tested. Of these, $31 \mathrm{CSF}$ and 15 sera were from proven cryptococcal cases and the remaining samples were from patients who did not have cryptococcosis (Figure 2).

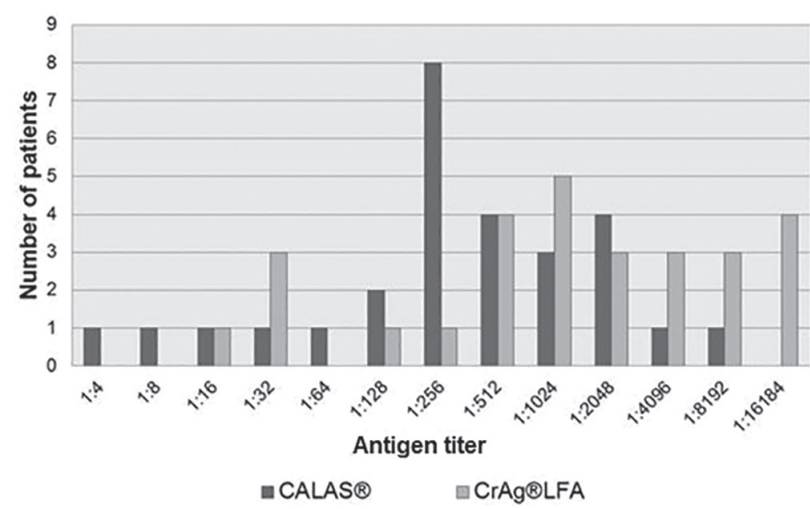

Figure 1 - Reactivity against Cryptococcus antigen: Comparison of the $\mathrm{CrAg}^{\circledR}$ LFA and the CALAS ${ }^{\circledR}$ tests. Analytical sensitivity of $\mathrm{CrAg}^{\circledR}$ LFA (light gray bars) and CALAS ${ }^{\circledR}$ (dark gray bars) are shown. The vertical axis shows the number of patients and the horizontal axis shows the final antigen titer

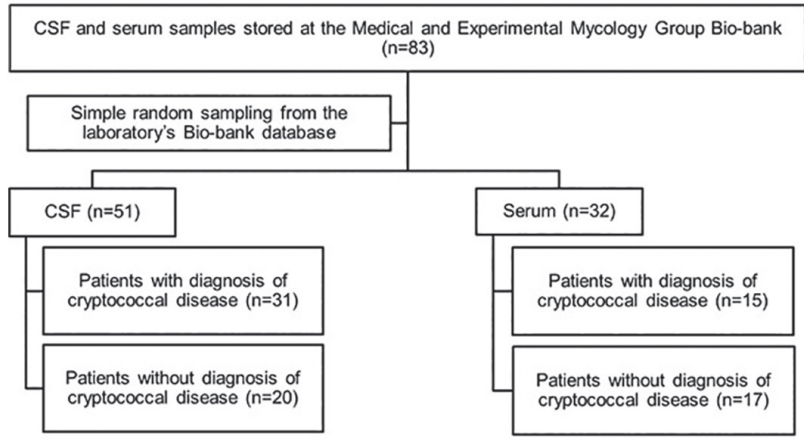

Figure 2 - Flowchart of serum and CSF samples analyzed during the validation of the $\mathrm{CrAg}^{\circledR}$ LFA. Study flowchart of analyzed samples stored at the Medical and Experimental Mycology Group biobank. A total of 83 samples were tested, corresponding to $51 \mathrm{CSF}$ and 32 sera
Sensitivity and specificity of $\mathrm{CrAg}^{\circledR}$ LFA in sera were both $100 \%$ (95\% confidence interval [95\% CI] $=97-100 \%$ ). For the CSF, both sensitivity and specificity were also $100 \%$ (95\% CI $=98-100 \%$ for sensitivity, and 95\% CI $=97-100 \%$ for specificity). In the two types of sample (serum and CSF), the concordance between $\mathrm{CrAg}^{\circledR}$ LFA and CALAS ${ }^{\circledR}$ was 1.0 (kappa index, 95\% CI = 1.0-1.0) (Table 1). When we compared the capacity to detect different Cryptococcus antigens levels between $\mathrm{CrAg}^{\circledR}$ LFA and $\mathrm{CALAS}^{\circledR}$ in CSF, $\mathrm{CrAg}^{\oplus}$ LFA demonstrated higher sensitivity to detect lower concentrations of antigen (Figure 1).

Results of this study showed that both serum and CSF specimens tested with the $\mathrm{CrAg}^{\circledR}$ LFA were $100 \%$ sensitive and specific. This is consistent with the reported sensitivity and specificity of this LFA in other laboratories around the world $^{6-10}$. The $\mathrm{CrAg}^{\circledR}$ LFA was also able to detect lower concentrations of antigens in CSF as compared with the $\mathrm{CALAS}^{\circledR}$ assay, a finding that has been previously reported ${ }^{9}$.

The $\mathrm{CrAg}^{\circledR}$ LFA test is a simple technique that can be easily performed in any laboratory; additionally, storage and incubation of reagents are performed at room temperature, and the specimen does not require pre-treatment with pronase or any other additional step.

A limitation of this study was that we were unable to access complete information from the clinical records on associated complications and mortality. Additionally, the number of samples that were used in this study was small. For the $\mathrm{CrAg}^{\circledR}$ LFA, two independent technicians performed the readings and no discordant results were found, although, for the latex agglutination test, only one technician with extensive training and expertise performed the readings. These diagnostic methods are supported by multiple validations with results similar to those observed in this study ${ }^{6-10}$.

In summary, the study presented here evaluated the $\mathrm{CrAg}^{\circledR}$ LFA test for detection of cryptococcosis using serum and CSF in a specialized laboratory in Medellín, Colombia. This test is relatively cheap and readily available, requires minimal laboratory steps and can be completed in less than 15 minutes using basic supplies. It shows high sensitivity, specificity, and concordance with currently $\mathrm{CrAg}$ tests used in the diagnosis of cryptococcosis. Additionally, the $\mathrm{CrAg}^{\circledR}$

Table 1 - Analysis of concordance between $\mathrm{CrAg}^{\circledR}$ LFA and CALAS ${ }^{\circledR}$

\begin{tabular}{|c|c|c|c|c|c|c|c|}
\hline \multicolumn{4}{|c|}{ A. Results in CSF samples $(n=51)$} & \multicolumn{4}{|c|}{ B. Results in serum samples $(n=32)$} \\
\hline & & \multicolumn{2}{|c|}{$\mathrm{CALAS}^{\circledR}$} & & & \multicolumn{2}{|c|}{$\mathrm{CALAS}^{\circledR}$} \\
\hline & & + & - & & & + & - \\
\hline \multirow{2}{*}{$\mathrm{CrAg}^{\circledR} \mathrm{LFA}$} & + & 31 & 0 & \multirow{2}{*}{$\mathrm{CrAg}^{\circledR} \mathrm{LFA}$} & + & 15 & 0 \\
\hline & - & 0 & 20 & & - & 0 & 17 \\
\hline \multicolumn{4}{|c|}{ kappa index $=1.0 ; 95 \% \mathrm{Cl}=1.0-1.0$} & \multicolumn{4}{|c|}{ kappa index $=1.0 ; 95 \% \mathrm{Cl}=1.0-1.0$} \\
\hline
\end{tabular}

CSF: cerebrospinal fluid, $95 \% \mathrm{Cl}$ : $95 \%$ confidence interval, +: positive, -: negative 
LFA demonstrated higher analytical sensitivity to detect low concentrations of cryptococcal antigen compared with the CALAS $^{\circledR}$ assay. This test could significantly reduce the time to diagnose and to treat cryptococcosis.

\section{ACKNOWLEDGMENTS}

The authors thank Catalina de Bedout, Karen Arango, Yorlady Valencia and Mike Sanchez from the laboratory diagnosis staff of the Medical Mycology laboratory at the Corporación para Investigaciones Biológicas (CIB), and Dr. Angela Restrepo and Dr. Oliver Clay for read the manuscript carefully as well as for their comments.

\section{CONFLICT OF INTERESTS}

The authors report no conflict of interests.

\section{FINANCIAL SUPPORT}

This work was supported by the Medical and Experimental Mycology Group, Corporación para Investigaciones Biológicas (CIB). $\mathrm{CrAg}^{\circledR}$ LFA reagents were donated by IMMY $^{\circledR}$, Norman, Oklahoma, USA. The findings and conclusions in this article are those of the authors and do not necessarily represent the views of the CDC.

\section{REFERENCES}

1. Park BJ, Wannemuehler KA, Marston BJ, Govender N, Pappas PG, Chiller TM. Estimation of the current global burden of cryptococcal meningitis among persons living with HIV/AIDS AIDS. 2009;23:525-30.

2. Kaplan JE, Vallabhaneni S, Smith RM, Chideya-Chihota S, Chehab J, Park B. Cryptococcal antigen screening and early antifungal treatment to prevent cryptococcal meningitis: a review of the literature. J Acquir Immune Defic Syndr. 2015;68 Suppl 3:331-9.
3. Pfeiffer CD, Wong B. Diagnostic immunology. In: Hospenthal DR, Rinaldi MG, editors. Diagnosis and treatment of human mycoses. $2^{\text {nd }}$ ed. New York: Humana Press; 2015. p.45-64.

4. De Pauw B, Walsh TJ, Donnelly JP, Stevens DA, Edwards JE, Calandra $\mathrm{T}$, et al. Revised definitions of invasive fungal disease from the European Organization for Research and Treatment of Cancer/Invasive Fungal Infections Cooperative Group and the National Institute of Allergy and Infectious Diseases Mycoses Study Group (EORTC/MSG) Consensus Group. Clin Infect Dis. 2008;46:1813-21.

5. Orozco LC. Medición en salud: diagnóstico y evaluación de resultados: un manual critico más allá de lo básico. Santander: Universidad Industrial de Santander; 2010. Validación de criterio o de la sensibilidad específica para predecir la calidad de las probabilidades; p.115-57.

6. Lindsley MD, Mekha N, Baggett HC, Surinthong Y, Autthateinchai $\mathrm{R}$, Sawatwong P, et al. Evaluation of a newly developed lateral flow immunoassay for the diagnosis of cryptococcosis. Clin Infect Dis. 2011;53:321-5.

7. Jarvis JN, Percival A, Bauman S, Pelfrey J, Meintjes G, Williams GN, et al Evaluation of a novel point-of-care cryptococcal antigen test on serum, plasma, and urine from patients with HIV-associated cryptococcal meningitis. Clin Infect Dis. 2011;53:1019-23.

8. McMullan BJ, Halliday C, Sorrell TC, Judd D, Sleiman S, Marriott $\mathrm{D}$, et al. Clinical utility of the cryptococcal antigen lateral flow assay in a diagnostic mycology laboratory. PLoS One. 2012;7:e49541.

9. Escandón P, Lizarazo J, Agudelo CI, Chiller T, Castañeda E. Evaluation of a rapid lateral flow immunoassay for the detection of cryptococcal antigen for the early diagnosis of cryptococcosis in HIV patients in Colombia. Med Mycol. 2013;51:765-8.

10. Huang HR, Fan LC, Rajbanshi B, Xu JF. Evaluation of a new cryptococcal antigen lateral flow immunoassay in serum, cerebrospinal fluid and urine for the diagnosis of cryptococcosis: a meta-analysis and systematic review. PLoS One. 2015;10:e0127117. 